\title{
THE 10 KEV INJECTOR FOR THE UNIVERSITY OF MARYLAND ELECTRON RING PROJECT*
}

\author{
T. Godlove, P. Haldemann, and D. Kehne, FM Technologies, Inc., S. Bernal, P. Chin, R. Kishek, \\ Y. Li, M. Reiser, M. Venturini, J.G. Wang, W.W. Zhang, Y. Zou, UMD, and I. Haber, NRL
}

\begin{abstract}
The $10 \mathrm{keV}, 100 \mathrm{~mA}$, Pierce-type electron gun and injector for the University of Maryland Electron Ring (UMER) Project are described. Using a pulsed control grid located $0.15 \mathrm{~mm}$ from the 4 -mm radius cathode, 100ns bunches are generated. The $\mathrm{A} / \mathrm{K}$ gap is variable, producing beam currents ranging from 50 to $160 \mathrm{~mA}$. A rotatable aperture plate is included to allow six different masks, including a pepperpot. Beam current after the aperture plate is measured with a built-in Rogowski coil. The injector line consists of a solenoid lens, five printedcircuit quadrupoles, and two in-line diagnostic chambers. A separate, multi-purpose chamber is placed at the end of the injector line during gun characterization studies for emittance, profile, and energy analysis.
\end{abstract}

\section{INTRODUCTION}

A model electron ring is being built at the University of Maryland to investigate the effects of space charge in bending systems, heavy-ion recirculators and rapidcycling rings with a limited number of turns [1]. For this research a space-charge-dominated, low emittance, relatively low energy (nonrelativistic) beam is desired. In this paper we describe the design of the injector, a key component of the electron ring. The injector consists of an electron gun, means for matching the beam into a quadrupole lattice, and pulsed elements for injecting the beam into the ring. The pulsed elements include a pulsed dipole and two Panofsky quadrupoles, one for the injected beam and the other for the recirculating beam [2].

The beam parameters were chosen to be $10 \mathrm{keV}$ and 100 $\mathrm{mA}$, corresponding to generalized perveance of 0.0015 , to model large accelerators with similar space-charge effects and to keep the cost in line with university research. Here the generalized perveance is $\mathrm{K}=2(\mathrm{I} / \mathrm{Io})(\mathrm{m} / \mathrm{M})(\beta \gamma)^{-3}$, where $\mathrm{I}$ is the beam current, $\mathrm{Io}=10^{7} \mathrm{mc} / \mathrm{e}=17 \mathrm{kA}, \mathrm{m}$ and $\mathrm{M}$ are the mass of an electron and the particle in question, respectively, and $\beta, \gamma$ are the usual normalized velocity and total energy of the particle. For $\mathrm{K}=0.0015$, the space charge forces are similar, for example, to a 100-MeV, 2.5 $\mathrm{kA}$ proton beam or a $1-\mathrm{GeV}, 5 \mathrm{kA}$ mass-200 beam. While such ion currents have not been achieved, nevertheless the space-charge forces put the $10-\mathrm{keV}$ beam in an important new regime of study which is important for a number of applications.

* Supported by the US Department of Energy.

\section{ELECTRON GUN}

The electron gun is similar to a $2.5 \mathrm{keV}$ gun used at the University of Maryland for some years [3]. It employs a conventional Pierce geometry and has a control grid located at $0.15 \mathrm{~mm}$ from an 8-mm diameter dispenser cathode. The electrode boundaries are shown in Fig. 1. While the gun is designed using EGN for an optimum current of $100 \mathrm{~mA}$, a variable $\mathrm{A} / \mathrm{K}$ gap is included to provide currents from 60 - to $140 \mathrm{~mA}$. A rotatable aperture plate, located near the beam waist, allows 6 different masks to be moved into the beamline.

The primary aperture, having a diameter twice that of the

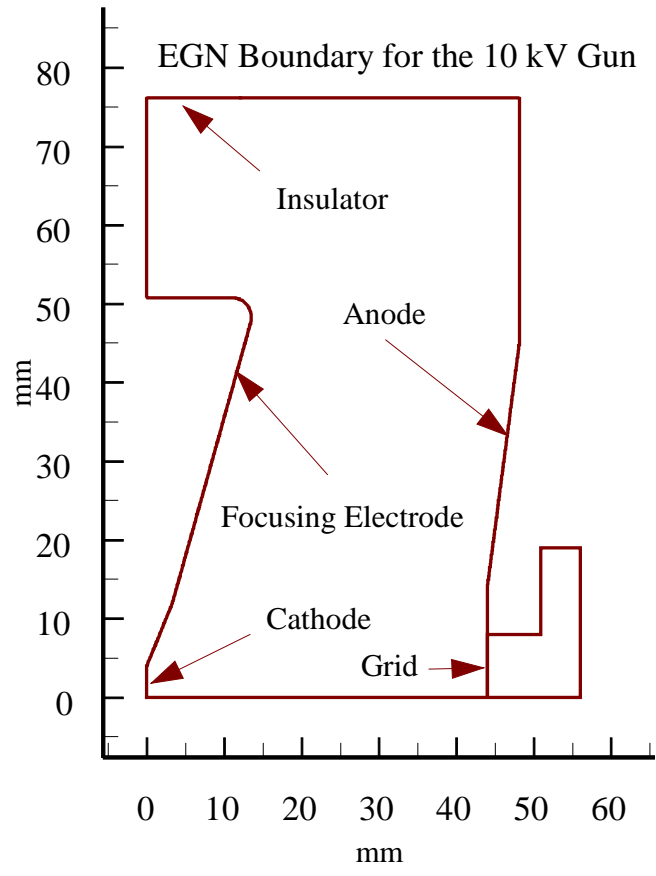

Fig. 1 Boundaries used in EGN gun design.

beam, allows full beam transport. Three round intercepting apertures provide currents of $0.5 \%, 15 \%$, and $55 \%$. A "pepperpot" mask is included for emittance measurements, and the final mask employs a 5-beamlet distribution to enable detailed comparison with PIC simulations. 


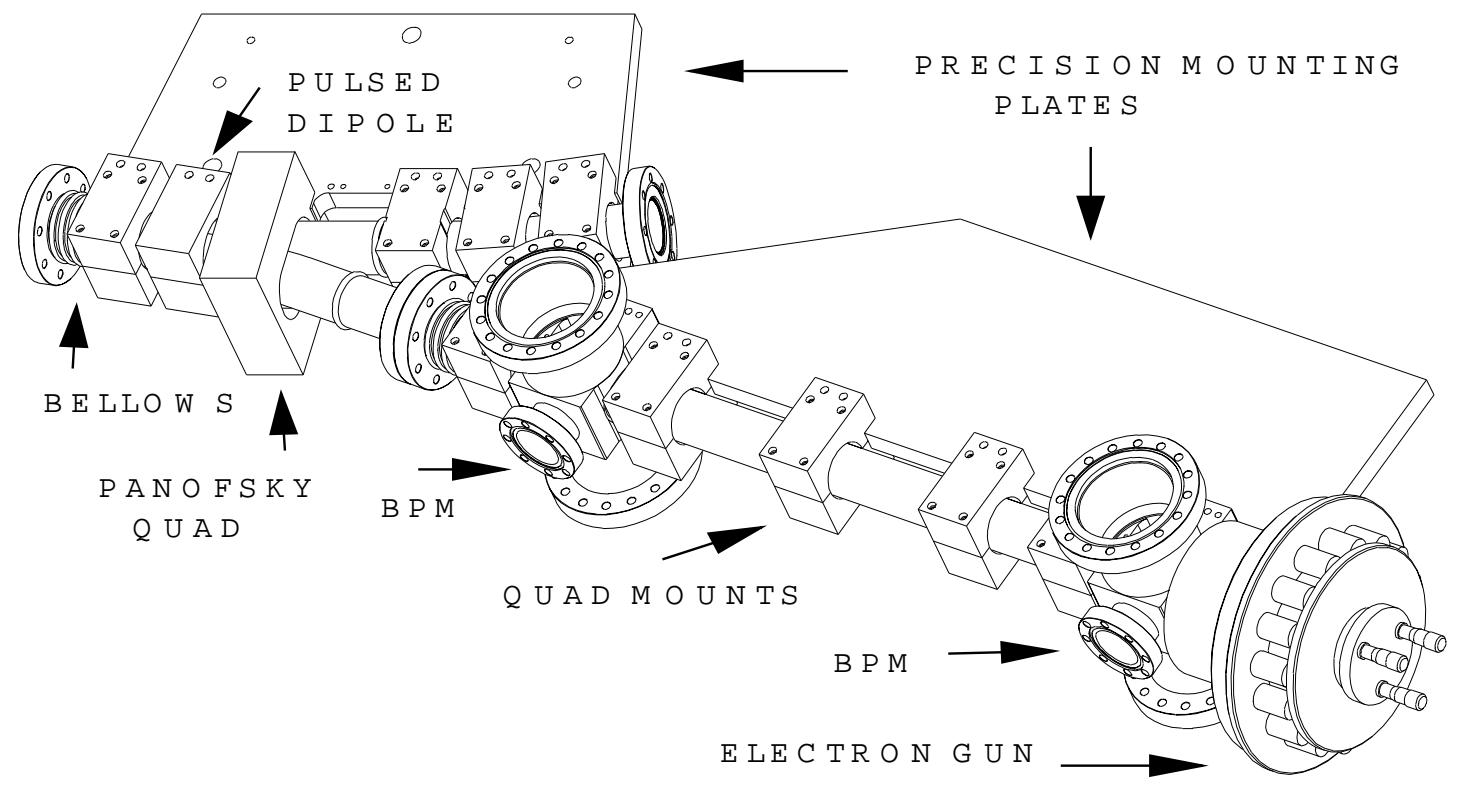

Fig. 2. Injector layout perspective.

Beam current is measured after the aperture plate with a built-in Rogowski coil. A gate valve is included to allow isolation from the remainder of the vacuum system. EGN simulations locate an $8-\mathrm{mm}$ diameter waist at $1.2 \mathrm{~cm}$ downstream from the anode grid. The $4 \mathrm{x}$ rms normalized thermal emittance of the cathode is calculated to be 3.5 mm-mrad. EGN predicts negligible growth above this. Analytic calculation of grid effects produces a total expected emittance of $4 \mathrm{~mm}$-mrad. While previous experience with the $2.5-\mathrm{keV}$ gun indicates emittance growth of up to $80 \%$ [3] above the thermal emittance, the specification for the $10-\mathrm{keV}$ injector allows for emittance growth of up to $280 \%$. This should be easily achievable.

The entire gun is bakeable to $250^{\circ} \mathrm{C}$. An 8 1/s ion pump mounted below the front face of the gun is sufficient. The field from the pump is $<0.1 \mathrm{G}$ at the cathode.

A large, multi-purpose, bakeable diagnostic chamber is under construction to characterize the electron gun both before and after it is mounted on the injector line.

Diagnostics include: (1) a fluorescent screen which is movable along the axis for transverse profile and envelope measurements; (2) a Faraday cup used as a beam stop and current monitor; (3) a slit-and-wire system for emittance measurements and (4) a retarding-field analyzer to measure the energy homogeneity. The last two diagnostics are mounted on flanges transverse to the beam axis so that they may be accurately inserted and retracted using remotely controlled precision motor drive.

Beam characterization will be performed in several steps: first with the gun alone, then with the injector line to study the lattice optics, then with dc injection elements, and finally with the pulsed dipole and Panofsky quadrupoles.

\section{INJECTOR LINE}

The injector line, shown in Fig. 2, has one solenoid and five printed-circuit quadrupoles [4] plus the Panofsky quadrupole. This enables matching the beam into the lattice as well as providing considerable freedom of adjustment of the parameters. Two diagnostic chambers are included in the injector line similar to those in the ring. Each houses a capacitive pickup beam position monitor (BPM), a fluorescent screen, and a 4-inch pumping port.

The injection angle is $10^{\circ}$, with the injection point located at one of the normal $10^{\circ}$ ring bends, enabling a $20^{\circ}$ angle between the two adjacent straight sections.

Because of the strong space charge, the quadrupoles in the ring are spaced with a half-lattice separation of $16 \mathrm{~cm}$. However, the cramped quarters near the injection point preclude such close spacing. For this reason the design uses two Panofsky quadrupoles (one shown in Fig. 2), one for the entering beam (which is turned off before the beam completes one turn) while the other serves as a normal ring quadrupole and must be turned on before the beam completes the first turn. With a circulation time of $200 \mathrm{~ns}$, the bunch length is expected to be 50-75 ns. The rise and fall time of the pulsed elements is expected be $\sim 30 \mathrm{~ns}$ [2]. Since the two Panofsky quadrupoles overlap slightly, the mutual interaction is of some concern and is being studied with bench models. 
The injection Y-shaped chamber will be glass, with a thin metallic coating on the inside to prevent charge buildup but allow fast field penetration.

Figure 3 shows a calculation using the K-V envelope code SPOT [5] based on a configuration without the bend. The position and strength of the solenoid and printed-circuit quadrupoles were adjusted based on the mechanical constraints noted above as well as optimum match to the FODO lattice. Orbit steering corrections will be accomplished with a combination of Helmholtz coils and thin discrete dipoles located in the injector line.

The 3-D PIC code, WARP [6], is being used for more detailed calculations of the beam dynamics, including the $10^{\circ}$ bends. Results to date indicate that emittance growth is reasonably small for 25 turns in the ring. One particularly sensitive tolerance is rotation of the printedcircuit quadrupoles about the beam axis. WARP calculations specific to the injector dynamics agree well with the SPOT results noted above, provided that initial runs were done to determine the optimum lattice conditions just before the inflector bend.

Transport of a 4-keV beam in a 1-m long FODO lattice with five printed-circuit quadrupoles has been studied recently [7]. Results indicate good agreement with both SPOT and WARP. This study provides an excellent foundation for the present design.

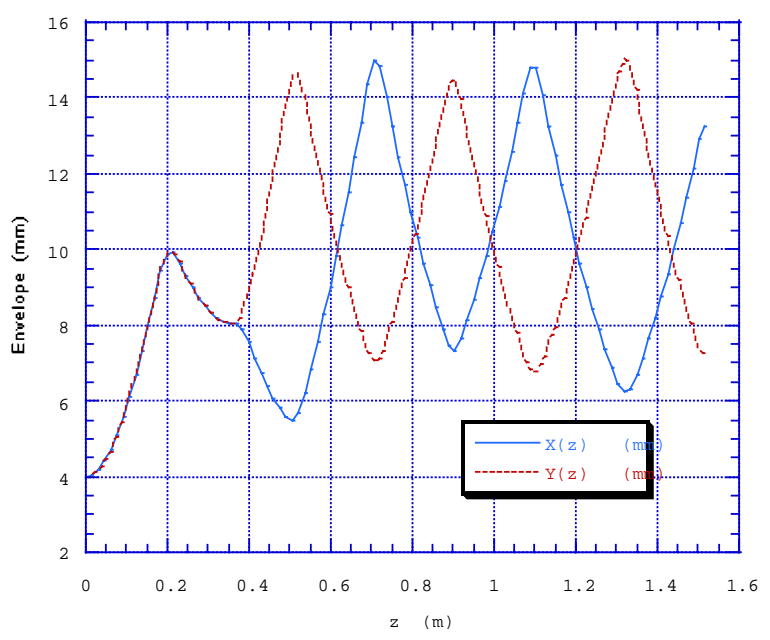

Fig. 3. Envelope calculation using SPOT.

\section{ELECTRONICS}

The pulser for the electron gun is similar to that used in the existing UMD $2.5-\mathrm{keV}$ gun. It consists of a highvoltage deck which houses a filament transformer, a grid bias supply, a 200-V pulser supply and the 100-ns pulse generator. The pulse generator uses a charged coaxial cable for the pulse network and a fast ( 1 ns) avalanche transistor switch.

Power is supplied through an isolation transformer, and pulses are supplied to the deck via a fiber-optic link. A trigger pulser has been built and tested which produces pulses from $1 \mathrm{~Hz}$ to $120 \mathrm{~Hz}$. The pulser is synchronized to the AC line and has delay capability up to $8 \mathrm{msec}$ for setting the trigger to coincide with zero AC fields at the cathode.

\section{CONCLUSION}

A complete injection system has been designed and is under construction for the University of Maryland electron ring. It is capable of delivering a high quality, matched beam to the ring with dynamics ranging from emittancedominated to space-charge-dominated, satisfying the needs of the experimental program. Initial tests of the gun are scheduled for the summer of 1999. Full installation and tests of the injector are planned to be completed by December 1999.

\section{REFERENCES}

1. M. Reiser et al, "The Maryland Electron Ring for Investigating Space-Charge Dominated Beams in a Circular FODO System" TUCR3, these proceedings.

2. Y. Li et al, "Design, Simulation and Test of Pulsed Panofsky Quadrupoles" THP169, these proceedings.

3. J.G. Wang, D.X. Wang and M. Reiser, Nucl. Inst. and Methods in Phys. Res. A316, 112 (1992).

4. T.F. Godlove, Santiago Bernal and Martin Reiser, Proc. 1995 Particle Accel. Conf., 2117 (1996).

5. C.K. Allen, S.K. Guharay, and M. Reiser, Proc. 1995 Particle Accel. Conf., 2324 (1996).

6. D.P. Grote et al, Fus. Engr. \& Des. 32-33, 193 (1996); R. Kishek et al, "Simulations of Collective Effects in the Space-Charge-Dominated Beam of the University of Maryland Electron Ring" TUP118, these proceedings; R. Kishek et al, "Effects of Quadrupole Rotations on the Transport of Space-Charge-Dominated Beams: Theory and Simulations Comparing Linacs with Circular Machines" TUP119, these proceedings.

7. S. Bernal et al, Phys. Rev. ST, Accel. \& Beams $\underline{4}$, 044202 (1998). 